\title{
Front Matter: Volume 10144
}

, "Front Matter: Volume 10144," Proc. SPIE 10144, Emerging Patterning Technologies, 1014401 (26 April 2017); doi: 10.1117/12.2279246

SPIE. Event: SPIE Advanced Lithography, 2017, San Jose, California, United States 


\title{
PROCEEDINGS OF SPIE
}

\section{Emerging Patterning Technologies}

\author{
Christopher Bencher \\ Editor
}

27 February - 1 March 2017

San Jose, California, United States

Cosponsored by

Tokyo Ohka Kogya America, Inc. (United States)

Published by

SPIE 
The papers in this volume were part of the technical conference cited on the cover and title page. Papers were selected and subject to review by the editors and conference program committee. Some conference presentations may not be available for publication. Additional papers and presentation recordings may be available online in the SPIE Digital Library at SPIEDigitallibrary.org.

The papers reflect the work and thoughts of the authors and are published herein as submitted. The publisher is not responsible for the validity of the information or for any outcomes resulting from reliance thereon.

Please use the following format to cite material from these proceedings:

Author(s), "Title of Paper," in Emerging Patterning Technologies, edited by Christopher Bencher, Proceedings of SPIE Vol. 10144 (SPIE, Bellingham, WA, 2017) Seven-digit Article CID Number.

ISSN: 0277-786X

ISSN: 1996-756X (electronic)

ISBN: 9781510607392

ISBN: 9781510607408 (electronic)

Published by

SPIE

P.O. Box 10, Bellingham, Washington 98227-0010 USA

Telephone +1 3606763290 (Pacific Time) · Fax +1 3606471445

SPIE.org

Copyright (C) 2017, Society of Photo-Optical Instrumentation Engineers.

Copying of material in this book for internal or personal use, or for the internal or personal use of specific clients, beyond the fair use provisions granted by the U.S. Copyright Law is authorized by SPIE subject to payment of copying fees. The Transactional Reporting Service base fee for this volume is $\$ 18.00$ per article (or portion thereof), which should be paid directly to the Copyright Clearance Center (CCC), 222 Rosewood Drive, Danvers, MA 01923. Payment may also be made electronically through CCC Online at copyright.com. Other copying for republication, resale, advertising or promotion, or any form of systematic or multiple reproduction of any material in this book is prohibited except with permission in writing from the publisher. The CCC fee code is $0277-786 \mathrm{X} / 17 / \$ 18.00$.

Printed in the United States of America.

Publication of record for individual papers is online in the SPIE Digital Library.

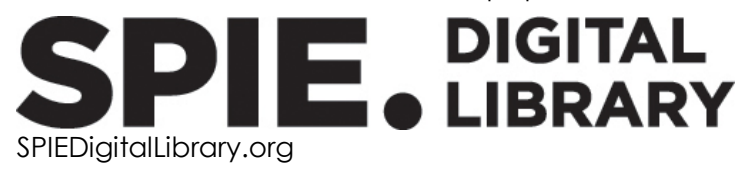

Paper Numbering: Proceedings of SPIE follow an e-First publication model. A unique citation identifier (CID) number is assigned to each article at the time of publication. Utilization of CIDs allows articles to be fully citable as soon as they are published online, and connects the same identifier to all online and print versions of the publication. SPIE uses a seven-digit CID article numbering system structured as follows:

- The first five digits correspond to the SPIE volume number.

- The last two digits indicate publication order within the volume using a Base 36 numbering

system employing both numerals and letters. These two-number sets start with 00, 01, 02, 03, 04, $05,06,07,08,09,0 A, O B \ldots$. OZ, followed by 10-1Z, 20-2Z, etc. The CID Number appears on each page of the manuscript. 


\title{
Contents
}

\author{
$\checkmark$ Authors \\ vii Conference Committee
}

\section{NANOPRINT LITHOGRAPHY FOR HIGH-VOLUME MANUFACTURING}

1014405 Nanoimprint system development for high-volume semiconductor manufacturing and the status of overlay performance (Invited Paper) [10144-4]

1014406 Study of nanoimprint lithography (NIL) for HVM of memory devices [10144-5]

1014407 Improved defectivity and particle control for nanoimprint lithography high-volume semiconductor manufacturing [10144-6]

1014408 High throughput nanoimprint lithography for semiconductor memory applications [10144-7]

1014409 Overlay control for nanoimprint lithography [10144-8]

\section{NANOPRINT MASKS AND APPLICATIONS}

10144 OB Selective surface smoothening of 3D micro-optical elements [10144-10]

\section{DSA INTEGRATION}

10144 OE Overview and development of EDA tools for integration of DSA into patterning solutions (Invited Paper) [10144-13]

10144 OF Free energy modeling of block-copolymer within pillar confinements on DSA lithography [10144-14]

10144 OG Process, design rule, and layout co-optimization for DSA based patterning of sub-10nm Finfet devices [10144-15]

DIRECT-WRITE, MASKLESS LITHOGRAPHY

$10144 \mathrm{OL}$ Simulation analysis of a miniaturized electron optics of the massively parallel electronbeam direct-write (MPEBDW) for multi-column system [10144-20]

DSA PROCESS AND INTEGRATION: JOINT SESSION WITH CONFERENCES 10146 AND 10144

1014400 Advanced surface affinity control for DSA contact hole shrink applications [10144-23] 
10144 OQ Pattern defect reduction and LER improvement of chemo-epitaxy DSA process [10144-25]

DSA MATERIALS AND PROCESSES: JOINT SESSION WITH CONFERENCES 10146 AND 10144

$10144 \mathrm{OR}$ Wide-range directed self-assembly lithography enabling wider range of applicable pattern size for both hexagonal multi-hole and line/space [10144-26]

POSTER SESSION

10144 OV Rules-based correction strategies setup on sub-micrometer line and space patterns for $200 \mathrm{~mm}$ wafer scale SmartNIL ${ }^{\mathrm{M}}$ process within an integration process flow [10144-30]

10144 OX RLT uniformity improvement utilizing multi-scale NIL process simulation [10144-32]

1014411 Latest evolution in a 300 mm graphoepitaxy pilot line flow for L/S applications [10144-36]

1014412 The opportunity and challenge of spin coat based nanoimprint lithography [10144-37]

1014413 Inspection and fabrication of nano-imprint stamp using electron and ion dual beam system [10144-38]

1014414 Model-based guiding pattern synthesis for on-target and robust assembly of via and contact layers using DSA [10144-39] 


\section{Authors}

Numbers in the index correspond to the last two digits of the seven-digit citation identifier (CID) article numbering system used in Proceedings of SPIE. The first five digits reflect the volume number. Base 36 numbering is employed for the last two digits and indicates the order of articles within the volume. Numbers start with 00, 01, 02, 03, 04, 05, 06, 07, 08, 09, 0A, 0B...0Z, followed by 10-1Z, 20-2Z, etc.

Aghili, Ali, 07

Ait-Ferhat, Dehia, OE

Altana, Mirco, OB

Argoud, Maxime, 00, 11

Asakawa, Koji, OR

Asano, Masafumi, 06

Azuma, Hisanobu, 07

Bok, Cheolkyu, 12

Bos, Sandra, 00

Brianceau, P., OV

Cayrefourcq, Ian, 00

Chamiot-Maitral, G., 11

Chevalier, Xavier, 00, 11

Chidambaram, Nachiappan, OB

Cho, Changhyun, OF

Cho, Jungbin, 12

Choi, Eun Hyuk, 06, 12

Choi, Jin, 05, 07

Chouiki, M., OV

Claveau, G., 11

Delachat, Florian, 00

Eibelhuber, M., OV

Esashi, Masayoshi, OL Fenger, Germain, OE

Fletcher, Brian, 08

Fukuhara, Kazuya, 06, 09

Gharbi, Ahmed, 00

Granik, Yuri, OE

Hatano, Masayuki, 06

Hayashi, Tatsuya, 05

Hazart, Jérôme, 00

Hiura, Mitsuru, 05

Hong, Hyeongsun, OF

Ido, Yasuyuki, $0 Q$

Ikegami, Naokatsu, OL

Im, Ji-Young, OX

Inanami, Ryoichi, OX

Irving, J. W., 08

Ito, Kiyohito, $0 Q$

Jones, Chris E., 07

Jung, ES, OF

Jung, Wooyung, 06, 09, 12

Kapral, Chris, OE

Khaira, Daman, OE

Khusnatdinov, Niyaz, 08

Kim, Hyun-Woo, OF

Kimura, Atsushi, 05

Kirchner, Robert, $\mathrm{OB}$

Kitano, Takahiro, $\mathrm{OQ}$
Kobayashi, Kei, 06, 12

Kobayashi, Sachiko, OX

Kodachi, Nobuhiro, 05

Kojima, Akira, OL

Komori, Motofumi, OX

Kono, Takuya, 06, 09, 0X, 12

Koshida, Nobuyoshi, OL

Krasnova, Polina, OE

Landis, S., OV

Lapeyre, Céline, 00

Laure, M., OV

Lee, Kyupil, OF

Lim, Yonghyun, 09, 12

Liu, Weijun, 08

Longsine, Whitney, 08

Ma, Yuansheng, OE, 14

Maeda, Shimon, OX

Matsuoka, Yoichi, 07

Mayr, M., OV

Mishima, Kazuhiko, 05

Mitra, Joydeep, OE, OG, 14

Mitsuyasu, Masaki, 09

Miyaguchi, Hiroshi, OL

Mizuno, Makoto, 07

Monget, Cédric, 00

Morikita, Shinya, $0 Q$

Morita, Seiji, OR

Morohoshi, Hiroshi, 05

Muramatsu, Makoto, OQ

Muroyama, Masanori, OL

Nakasugi, Tetsuro, 06, 09, 12

Nakayama, Takahiro, 07

Navarro, Christophe, 00, 11

Nicolet, Célia, 00

Nishi, Takanori, $0 Q$

Oikawa, Noriaki, $0 Q$

Pain, Laurent, 00, 0V

Pan, David Z., OG, 14

Park, Jemin, OF

Park, Joonsoo, OF

Park, Seokhan, OF

Pimenta Barros, Patricia, 00, 11

Saito, Ryuichi, OR

Saito, Yusuke, $0 Q$

Sasao, Norikatsu, OR

Sawabe, Tomoaki, OR

Schift, Helmut, OB

Shy, Shyi-Long, 13

Stachowiak, Tim, 08 
Suda, Ryutaro, OL

Sugimura, Shinobu, OR

Suzaki, Yoshio, 05

Suzuki, Masato, 06, 09

Takabayashi, Yukio, 05, 07

Teyssedre, H., OV

Thanner, C., OV

Ting, Yung-Chiang, 13

Tiron, Raluca, 00, 11

Tobana, Toshikatsu, $0 Q$

Tokue, Hiroshi, 06

Torres, J. Andres, OE, OG, 14

Totsu, Kentaro, OL

Traub, Matthew, 08

Tsuda, Hirotaka, OX

Tsuji, Masatoshi, 12

Washida, Kazuhiro, OX

Wimplinger, M., OV

Yamamoto, Ryosuke, OR

Yamashita, Kyoji, OX

Ye, Zhengmao, 08

Yonekawa, Masami, 07

Yoshida, Shinya, OL

Yoshida, Takahiro, 05

Yoshida, Takashi, OL

You, Gen, OQ

Zhang, Wei, 08

Zorbach, W., OV 


\section{Conference Committee}

Symposium Chair

Bruce W. Smith, Rochester Institute of Technology (United States)

Symposium Co-Chair

Will Conley, Cymer, An ASML Company (United States)

Conference Chair

Christopher Bencher, Applied Materials, Inc. (United States)

Conference Co-Chair

Joy Y. Cheng, Taiwan Semiconductor Manufacturing Company (Taiwan)

Conference Program Committee

Alan D. Brodie, KLA-Tencor Corporation (United States)

Kenneth R. Carter, University of Massachusetts Amherst (United States)

Damon M. Cole, Intel Corporation (United States)

Juan J. de Pablo, The University of Chicago (United States)

Michael A. Guillorn, IBM Thomas J. Watson Research Center (United States)

Naoya Hayashi, Dai Nippon Printing Company, Ltd. (Japan)

Daniel J. C. Herr, The University of North Carolina at Greensboro

(United States)

Tatsuhiko Higashiki, Toshiba Corporation (Japan)

James A. Liddle, National Institute of Standards and Technology

(United States)

Shy-Jay Lin, Taiwan Semiconductor Manufacturing Company Ltd.

(Taiwan)

Chi-Chun Liu, IBM Corporation (United States)

Hans Loeschner, IMS Nanofabrication AG (Austria)

John G. Maltabes, Hewlett-Packard Laboratories (United States)

Dan B. Millward, Micron Technology, Inc. (United States)

Laurent Pain, CEA-LETI (France)

Moshe E. Preil, KLA-Tencor Corporation (United States)

Ivo W. Rangelow, Technische Universität Ilmenau (Germany)

Benjamen M. Rathsack, Tokyo Electron America, Inc. (United States)

Douglas J. Resnick, Canon Nanotechnologies, Inc. (United States)

Ricardo Ruiz, HGST (United States)

Frank M. Schellenberg, Consultant (United States)

Helmut Schift, Paul Scherrer Institut (Switzerland) 
Ines A. Stolberg, Vistec Electron Beam Lithography Group (Germany) Hsinyu Tsai, IBM Thomas J. Watson Research Center (United States)

Kevin T. Turner, University of Pennsylvania (United States)

Marco J. Wieland, MAPPER Lithography (Netherlands)

Wei Wu, The University of Southern California (United States)

\section{Session Chairs}

1 Keynote Session

Christopher Bencher, Applied Materials, Inc. (United States)

Hans Loescher, IMS Nanofabrications AG (Austria)

2 Nanoprint Lithography for High-Volume Manufacturing

Douglas J. Resnick, Canon Nanotechnologies, Inc. (United States)

John G. Maltabes, Jordan Valley Semiconductors, Inc. (United States)

3 Nanoprint Masks and Applications

Naoya Hayashi, Dai Nippon Printing Company, Ltd. (Japan)

Helmut Schift, Paul Scherrer Institut (Switzerland)

4 DSA Integration

James A. Liddle, National Institute of Standards and Technology (United States)

Hsinyu Tsai, IBM Thomas J. Watson Research Center (United States)

5 Direct-Write, Maskless Lithography

Ines A. Stolberg, Vistec Electron Beam GmbH (Germany)

Marco Wieland, MAPPER Lithography (Netherlands)

6 DSA Process and Integration: Joint Session with Conferences 10146

and 10144

Ralph R. Dammel, EMD Performance Materials Corporation

(United States)

Chi-Chun Liu, IBM Corporation (United States)

7 DSA Materials and Processes: Joint Session with Conferences 10146

and 10144

Daniel P. Sanders, IBM Research - Almaden (United States)

Benjamen M. Rathsack, Tokyo Electron America, Inc. (United States) 